\title{
Microstrip Bandpass Filters With Dissimilar Resonators for Suppression of Spurious Responses
}

\author{
Pu-Hua Deng, Shih-Cheng Lin, Yo-Shen Lin, Chi-Hsueh Wang, and Chun Hsiung Chen \\ Department of Electrical Engineering and Graduate Institute of Communication Engineering, \\ National Taiwan University, Taipei 106, Taiwan
}

TEL : 886-2-23635251-ext.221, Fax : 886-2-23638247

E-MAIL:

\begin{abstract}
Microstrip bandpass filters with an extended rejection bandwidth are proposed, using dissimilar resonators for spurious suppression. The combination of dissimilar resonator structures with same fundamental frequency but with different spurious frequencies may improve the stopband rejection of a bandpass filter by the partially mutual cancellation of spurious responses. Specifically, a 4th-order coupled-resonator microstrip bandpass filter, centered at $\mathrm{f}_{0}=1.97 \mathrm{GHz}$, with better than $21.48 \mathrm{~dB}$ stopband rejection up to $9.72 \mathrm{GHz}\left(4.93 \mathrm{f}_{0}\right)$ is implemented and investigated.
\end{abstract}

\section{INTRODUCTION}

In microwave communication systems, filters with good selectivity are required to enhance the system performance. Recently, several filter structures using halfwavelength $(\lambda / 2)$ resonators have been proposed [1]-[3]. These filters possess multiple transmission zeros such that better selectivity can be achieved. In order to reduce interference by keeping the out-band signals from reaching a sensitive receiver, a high performance filter with wider upper stopband is also required. However, the planar bandpass filters made of $\lambda / 2$ uniform-impedance resonators (UIRs) have inherently spurious passbands at the multiples of center frequency $\mathrm{f}_{0}$. Several filters using the stepped-impedance resonators (SIRs) were presented to shift the spurious passbands to higher frequencies so as to extend the upper rejection band [2]-[4]. However, the stepped-impedance method can only push the first spurious passband to the higher frequency instead of suppressing it. If one would like to move the first spurious passband to $4 f_{0}$ by using a $\lambda / 2$ SIR, a large impedance ratio for the SIR is required such that the layout of the filter becomes difficult due to the fabrication limit.

To solve these drawbacks, several methods to achieve spurious passband rejection have been proposed [5]-[7]. In [6], by controlling the locations of input/output tapping points of a SIR filter, the spurious passband was cancelled by a notch inserted at the spurious frequency. But two additional quarter-wavelength $(\lambda / 4)$ transformers are required at the input and output, thereby increasing the circuit area.

In this study, a simple and effective method for suppressing the spurious passbands of a bandpass filter is

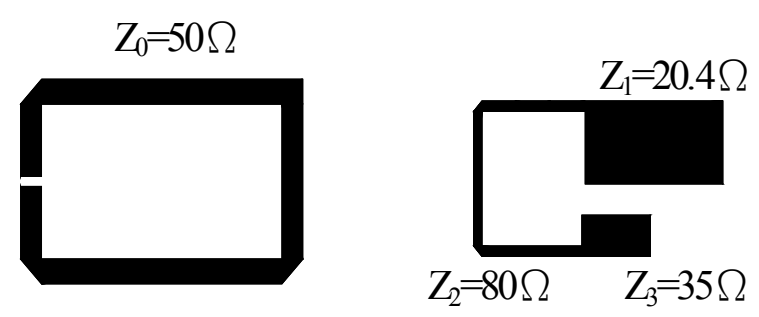

(a)

(b)

Fig. 1 (a) $\lambda / 2$ uniform-impedance resonator (UIR) and (b) $\lambda / 2$ stepped-impedance resonator (SIR).

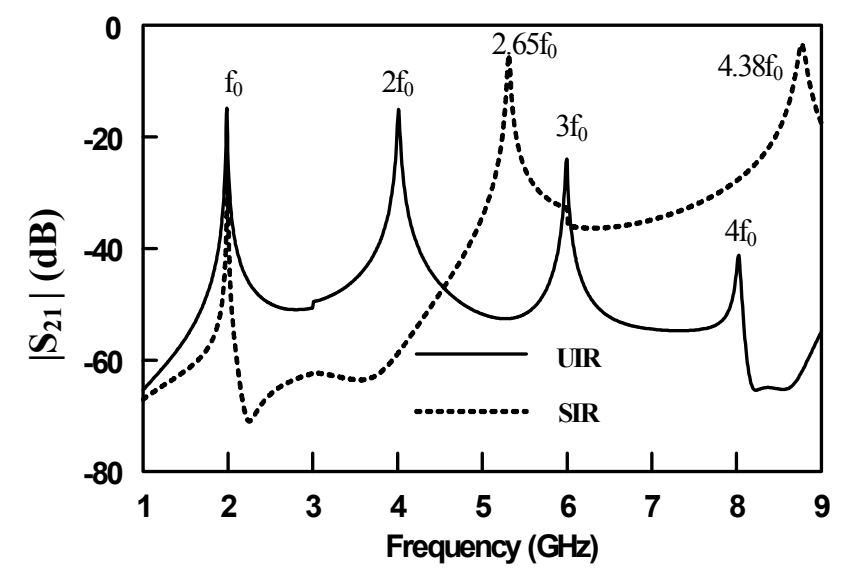

Fig. 2. Frequency responses of UIR and SIR in Fig. 1.

proposed. By incorporating resonators that have same fundamental resonant frequency but with different spurious frequencies in the filter structure, the spurious responses can be suppressed without increasing the circuit area and circuit element. Based on this approach, a coupled-resonator microstrip bandpass filter with good selectivity and wide rejection band is demonstrated.

\section{BASIC $\lambda / 2$ RESONATORS}

Fig. 1(a) shows a $\lambda / 2$ uniform-impedance resonator (UIR) that is commonly used in bandpass filter designs. The fundamental resonant frequency is located at $\mathrm{f}_{0}$. As shown in Fig. 2, its first, second, and third spurious resonances are found at $2 \mathrm{f}_{0}, 3 \mathrm{f}_{0}$, and $4 \mathrm{f}_{0}$, respectively. 


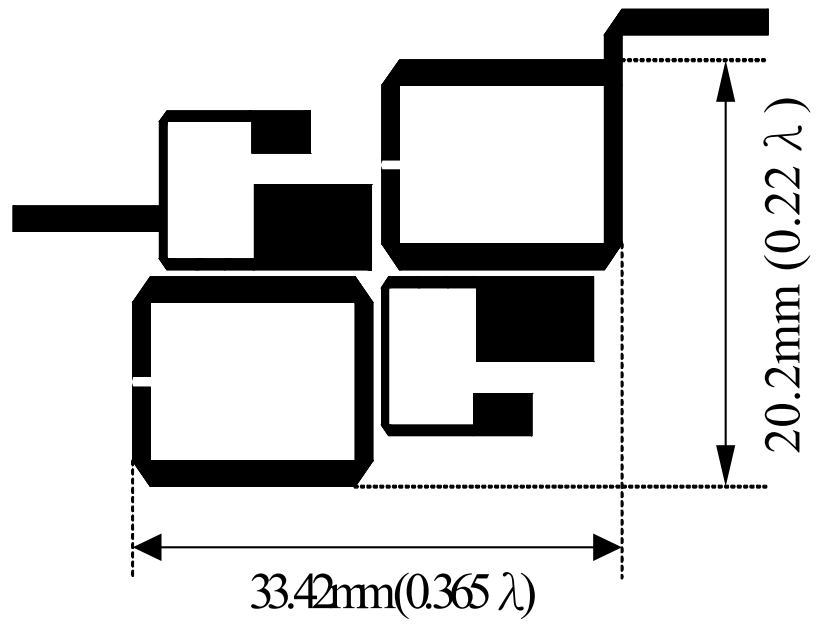

Fig. 3. Layout of the proposed 4th-order coupled-resonator microstrip bandpass filter composed of two kinds of resonators.

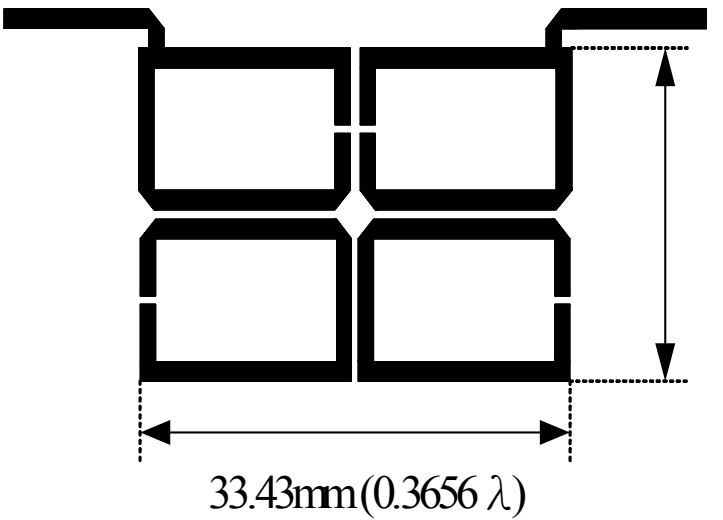

Fig. 4. Layout of the conventional 4th-order coupled-resonator microstrip filter based on identical $\lambda / 2$ uniform-impedance resonators.

As a result, a bandpass filter based on the UIR in Fig. 1(a) will exhibit spurious passbands at these frequencies. Fig. 1(b) shows a $\lambda / 2$ SIR that consists of three segments of transmission lines with different characteristic impedances $Z_{1}, Z_{2}$, and $Z_{3}$. By properly adjusting the impedance ratio of SIR, the spurious passbands can be pushed to higher frequencies [8]. For the SIR in Fig. 1(b), the fundamental resonant frequency is also designed at $f_{0}$ whereas the first and second spurious resonances are located at $2.65 \mathrm{f}_{0}$ and $4.38 \mathrm{f}_{0}$ as shown in Fig. 2. Note that the two resonators in Fig. 1(a) and Fig. 1(b) exhibit the same fundamental resonant frequency $\mathrm{f}_{0}$ but different spurious frequencies. Hence by combining them properly in a filter structure one may lead to the mutual suppression of spurious passbands and thereby achieves a better stopband rejection.

\section{FILTER STRUCTURES}

Shown in Fig. 3 is the layout of the proposed 4th-order coupled-resonator microstrip bandpass filter that utilizes

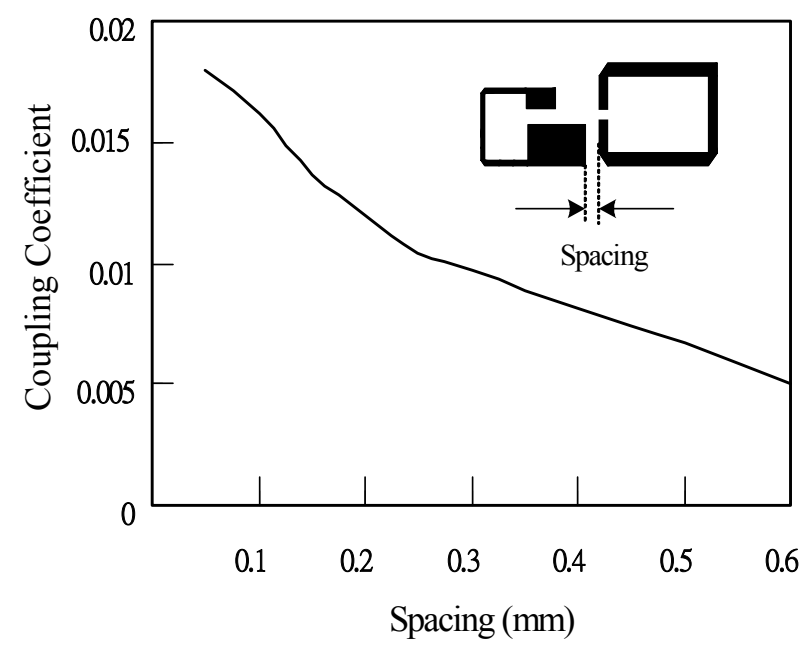

(a)

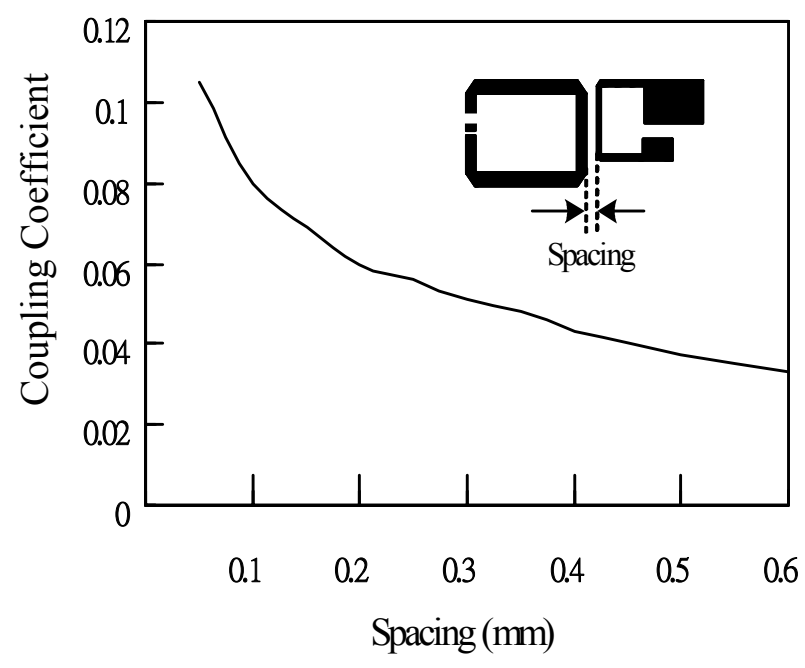

(b)

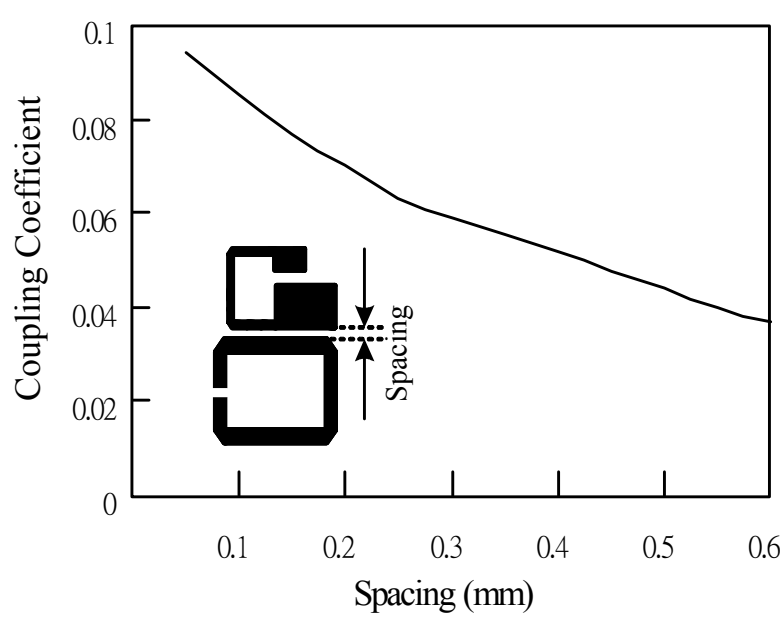

(c)

Fig. 5. Coupling structures and design curves involving coupling coefficients for: (a) electric coupling, (b) magnetic coupling, and (c) mixed coupling. 


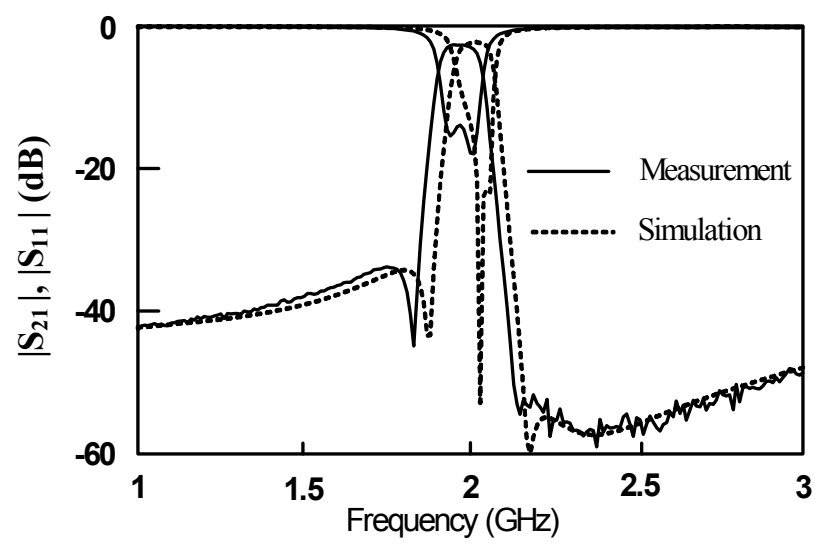

(a)

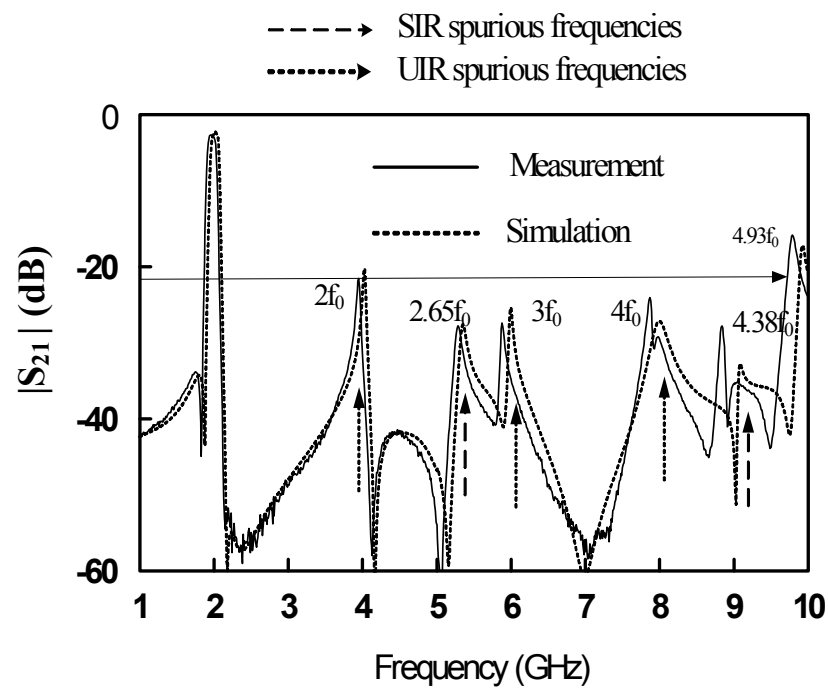

(b)

Fig. 6. Measured and simulated results of the proposed 4thorder microstrip filter in Fig. 3. (a) Narrowband and (b) wideband frequency responses.

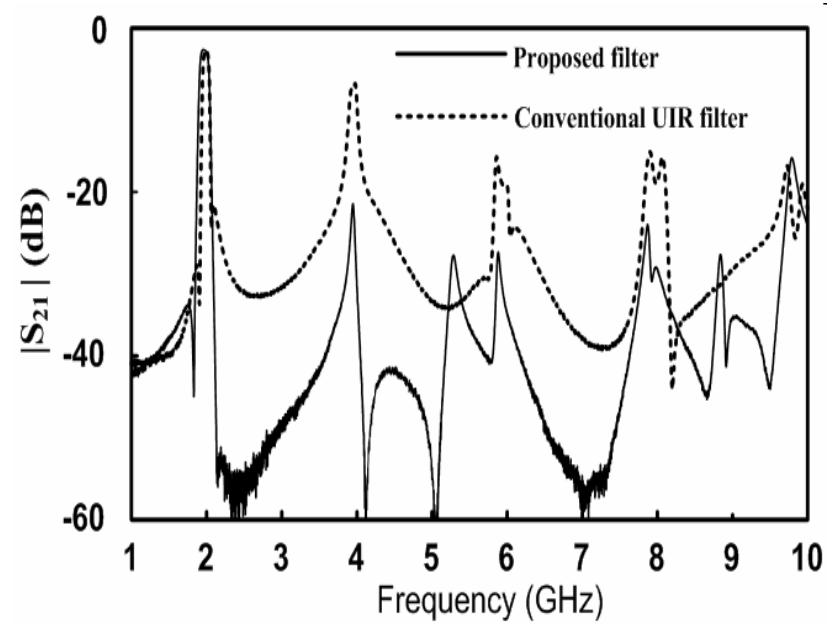

Fig. 7. Measured $\left|S_{21}\right|$ of the proposed filter (Fig. 3) and the conventional filter with idential resonators (Fig.5). the two kinds of resonators in Fig. 1(a) and Fig. 1(b) The four resonators are arranged like the conventional coupled-resonator filter in Fig. 4, such that two transmission zeros can be achieved to improve the selectivity [1].

Fig. 5 shows the three basic coupling structures and the related design curves for the filter in Fig.3. The structure in Fig. 5(a) is an electric coupling one because the electric field is stronger near the open end of the resonator. By similar reasoning, the structure in Fig. 5 (b) provides a magnetic coupling because the magnetic field is stronger near the center of the resonator. The structure in Fig. 5(c) provides both electric and magnetic couplings. The design curves involving coupling coefficients are obtained from the two calculated resonant frequencies associated with the coupled structures [9]. These curves are useful in determining the proper spacings to produce the required coupling coefficients in the design phase. The external quality factor $\mathrm{Q}_{\mathrm{e}}$ for the tapped input can also be extracted by the method in [9].

For the specifications with the center frequency at 2 $\mathrm{GHz}$ and $6.5 \%$ bandwidth, the coupling coefficients and the loaded $\mathrm{Q}_{\mathrm{e}}$ of the proposed filter can be found as

$$
\begin{aligned}
& K=\left[\begin{array}{lccc}
0 & 0.056 & 0 & -0.0055 \\
0.056 & 0 & 0.035 & 0 \\
0 & 0.035 & 0 & 0.056 \\
-0.0055 & 0 & 0.056 & 0
\end{array}\right] \\
& Q_{e}=12.9 .
\end{aligned}
$$

The proposed filter structure in Fig. 3 is fabricated on a Rogers RO4003C substrate $\left(\varepsilon_{\mathrm{r}}=3.38, \tan \delta=0.0027\right.$, and thickness $\mathrm{h}=0.508 \mathrm{~mm}$ ). This filter has a dimension of $0.365 \lambda \times 0.22 \lambda(33.42 \mathrm{~mm} \times 20.2 \mathrm{~mm})$, where $\lambda$ is the guided wavelength of the microstrip structure at $\mathrm{f}_{0}$.

The measured and simulated results are shown in Fig. 6. The simulation is done by the full-wave simulator Ansoft Ensemble. The measured center frequency is at $1.97 \mathrm{GHz}$. The measured minimum insertion loss is $2.68 \mathrm{~dB}$ at 1.97 $\mathrm{GHz}$, and the $3-\mathrm{dB}$ bandwidth is $6.7 \%$. As shown in Fig. 6(b), the stopband is extended to $9.72 \mathrm{GHz}\left(4.93 \mathrm{f}_{0}\right)$ with better than $21.48 \mathrm{~dB}$ rejection. The spurious resonant frequencies of the SIR and UIR adopted for this filter are also labeled in Fig. 6(b). Due to the employment of two kinds of resonators with different spurious frequencies, no strong resonance in the filter structure would occur at these spurious frequencies. Therefore, a better stopband rejection is obtained.

The spurious suppression for the proposed filter is also compared to that of the conventional one with identical resonators (Fig. 4). As shown in Fig. 7, much better stopband rejection is observed as expected.

\section{CONCLUSION}

In this paper, a method of extending the rejection bandwidth of bandpass filter is proposed. By utilizing dissimilar resonators with same fundamental resonant 
frequency but with different spurious frequencies in the filter structure, the spurious suppression can be enhanced and a wide rejection band can be obtained. Specifically, a 4th-order coupled-resonator microstrip bandpass filter composed of two kinds of $\lambda / 2$ resonators is designed and investigated. The stopband is extended to $9.72 \mathrm{GHz}$ $\left(4.93 \mathrm{f}_{0}\right)$ with more than $21.48 \mathrm{~dB}$ rejection. The measured results demonstrate that the proposed method can effectively improve the stopband performance.

\section{ACKNOWLEDGEMENT}

This work was supported by the National Science Council of Taiwan under Grant NSC 93-2752-E-002-001PAE and Grant NSC 93-2219-E-002-021.

\section{REFERENCES}

[1] J. S. Hong and M. J. Lancaster, "Couplings of microstrip square open-loop resonators for cross-coupled planar microwave filters," IEEE Trans. Microwave Theory Tech., vol. 44, pp. 2099 - 2109, Nov. 1996.

[2] S.-Y. Lee and C.-M. Tsai, "New cross-coupled filter design using improved hairpin resonators," IEEE Trans. Microwave Theory Tech., vol. 48, pp. 2482-2490, Dec. 2000.

[3] C.-M. Tsai, S.-Y. Lee, and C.-C. Tsai, "Performance of a planar filter using a $0^{\circ}$ feed strucure," IEEE Trans.
Microwave Theory Tech., vol. 50, pp. 2362-2367, Oct. 2002.

[4] M. Sagawa, K. Takahashi, and M. Makimoto, "Miniaturized hairpin resonator filters and their application to receiver front-end MIC's," IEEE Trans. Microwave Theory Tech., vol. 37, pp. 1991-1997, Dec.1989 .

[5] T. Lopetegi, M. A. G. Laso, J. Hernandez, M. Bacaicoa, D. Benito, M. J. Grade, M. Sorolla, and M. Guglielmi, "New microstrip "Wiggly-Line" filters with spurious passband suppression," IEEE Trans. Microwave Theory Tech., vol. 49, pp. 1593-1598, Sept. 2001.

[6] J.-T. Kuo and E. Shih, "Microstrip stepped impedance resonator bandpass filter with an extended optimal rejection bandwidth," IEEE Trans. Microwave Theory Tech., vol. 51, pp. 1554-1559, May 2003.

[7] S.-M. Wang, C.-H. Chi, M.-Y. Hsieh, and C.-Y. Chang, "Miniaturized spurious passband suppression microstrip filter using meandered parallel coupled lines," IEEE Trans. Microwave Theory Tech., vol. 53, pp. 747-753, Feb. 2005.

[8] M. Sagawa, M. Makimoto, and S. Yamashita, "Geometrical structures and fundamental characteristics of microwave stepped-impedance resonators," IEEE Trans. Microwave Theory Tech., vol. 45, pp. 1078 - 1085, July 1997.

[9] J. S. Hong and M. J. Lancaster, Microstrip Filters for RF/Microwave Applications. New York: Wiley, 2001. 